\section{A New Method of Measuring Nuclear Magnetic Moment*}

It is the purpose of this note to describe an experiment in which nuclear magnetic moment is measured very directly. The method is capable of very high precision and extension to a large number and variety of nuclei.

Consider a beam of molecules, such as $\mathrm{LiCl}$, traversing a magnetic field which is sufficiently strong to decouple completely the nuclear spins from one another and from the molecular rotation. If a small oscillating magnetic field is applied at right angles to a much larger constant field, a re-orientation of the nuclear spin and magnetic moment with respect to the constant field will occur when the frequency of the oscillating field is close to the Larmor frequency of precession of the particular angular momentum vector in question. This precession frequency is given by

$$
v=\mu H / h i=g(i) \mu_{0} H / h .
$$

To apply these ideas a beam of molecules in a $1 \boldsymbol{\Sigma}$ state (no electronic moment) is spread by an inhomogeneous magnetic field and refocused onto a detector by a subsequent field, somewhat as in the experiment of Kellogg, Rabi and Zacharias. ${ }^{1}$ As in that experiment the re-orienting field is placed in the region between the two magnets. The homogeneous field is produced by an electromagnet capable of supplying uniform fields up to 6000 gauss in a gap $6 \mathrm{~mm}$ wide and $5 \mathrm{~cm}$ long. In the gap is placed a loop of wire in the form of a hairpin (with its axis parallel to the direction of the beam) which is connected to a source of current at radiofrequency to produce the oscillating field at right angles to the steady field. If a re-orientation of a spin occurs in this field, the subsequent conditions in the second deflecting field are no longer correct for refocusing, and the intensity at the detector goes down. The experimental procedure is to vary the homogeneous

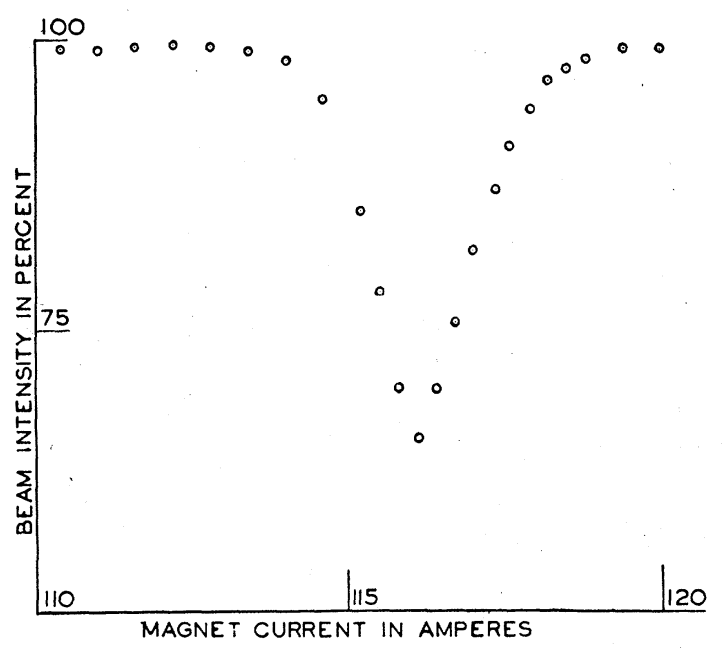

FIG. 1. Curve showing refocused beam intensity at various values of the homogeneous field. One ampere corresponds to about 18.4 gauss cycles per second. field for some given value of the frequency of the oscillating field until the resonance is observed by a drop in intensity at the detector and a subsequent recovery when the resonance value is passed.

The re-orientation process is more accurately described as one in which transitions occur between the various magnetic levels given by the quantum number $m_{i}$ of the particular angular momentum vector in question. An exact solution for the transition probability was given by $\mathrm{Rabi}^{2,3}$ for the case where the variable field rotates rather than oscillates. However, it is more convenient experimentally to use an oscillating field, in which case the transition probability is approximately the same for weak oscillating fields near the resonance frequency, except that $\vartheta$ is replaced by $\vartheta / 2$ in Eq. (13). With this replacement and with passage to the limit of weak oscillating fields, the formula becomes for the case of $i=\frac{1}{2}$

$$
P\left(\frac{1}{2},-\frac{1}{2}\right)=\frac{\vartheta^{2}}{(1-q)^{2}+q \vartheta^{2}} \sin ^{2}\left\{\pi \operatorname{tr}\left[(1-q)^{2}+q \vartheta^{2}\right]^{\frac{1}{2}}\right\},
$$

where $\vartheta$ is $\frac{1}{2}$ the ratio of the oscillating field to the steady field, $q$ is the ratio of the Larmor frequency of Eq. (1) to the frequency $r$ of the oscillating field. The derominator of the expression is the familiar resonance denominator. The formula is generalized to any spin $i$ by formula (17). ${ }^{2}$

In the theory of this experiment, $t$, in Eq. (2), is replaced by $L / v$, where $L$ is the length of the oscillating region of the field, and $v$ is the molecular velocity. $P\left(\frac{1}{2},-\frac{1}{2}\right)$ must then be averaged over the Maxwellian distribution of velocities. However, the first term is not affected by the velocity distribution if $t$ is long enough for many oscillations to take place. The average value of the $\sin ^{2}$ term over the velocity distribution is approximately $\frac{1}{2}$.

To produce deflections of the weakly magnetic molecules sufficient to make the apparatus sensitive to this effect, the beam is made $245 \mathrm{~cm}$ long; the first deflecting field is $52 \mathrm{~cm}$ in length and the second $100 \mathrm{~cm}$.

We have tried this experiment with $\mathrm{LiCl}$ and observed the resonance peaks of $\mathrm{Li}$ and $\mathrm{Cl}$. The effects are very striking and the resonances sharp (Fig. 1). A full account of this experiment, together with the values of the nuclear moments, will be published when the homogeneous field is recalibrated.

\section{I. RABI \\ J. R. ZACHARIAS \\ S. Millman \\ P. Kusch}

Hunter College (J. R. Z.),
Columbia University,
New York, N. Y.

January $31,1938$.

* Publication assisted by the Ernest Kempton Adams Fund for Physical Research of Columbia University.

1 Kellogg, Rabi and Zacharias, Phys. Rev. 50, 472 (1936).

2 Rabi, Phys. Rev. 51, 652 (1937).

${ }^{3}$ C. J. Gorter, Physica 9,995 (1936). We are very much indebted to Dr. Gorter who, when visiting our laboratory in September 1937, drew measure nuclear moments by observing the rise in temperature of solids measure nuclear moments by observing the rise in temperature of solids
placed in a constant magnetic field on which an oscillating field was
superimposed. Dr. F. Bloch has independently worked out similar ideas but for another purpose (unpublished). 